\title{
Violencia de pareja en estudiantes universitarios del sur de Chile*
}

\section{Intimate Partner Violence in South Chilean College Students}

Recibido: junio 5 de 2009 ｜ Revisado: noviembre 26 de 2009 | Aceptado: febrero 14 de 2010

\author{
María BEATRIZ VizCARRA LARRAÑAGA** \\ ANA MARÍA PÓO FigueroA ${ }^{* * *}$ \\ Universidad de la Frontera, Temuco, Chile
}

Para citar este artículo. Vizcarra, M. B., \& Póo, A. M. (2011). Violencia de pareja en estudiantes universitarios del sur de Chile. Universitas Psychologica, 10(1), 89-98.

Agradecimientos: Esta investigación fue financiada por la Dirección de Investigación de la Universidad de La Frontera. Proyecto Didufro 120447, 2004.

** Departamento de Psicología Universidad de La Frontera. Psicóloga, Profesor Asociado. Dirección Postal: Francisco Salazar 01145 Temuco., Chile. Dirección Electrónica: vizcarra@ufro.cl

**** Departamento de Psicología de la Universidad de la Frontera. Psicóloga-Matrona, Profesor Asistente. Dirección Postal: Francisco Salazar 01145 Temuco, Chile. Dirección Electrónica: ampoo@ufro.cl

\section{RES UMEN}

El presente estudio tuvo como objetivos estimar la magnitud de la violencia de pareja en estudiantes universitarios en la Región sur de Chile, describir las formas en que se manifiesta; los factores de riesgo asociados, las consecuencias y las estrategias utilizadas para enfrentarla. Se utilizó un diseño cuantitativo descriptivo. La muestra estuvo constituida por 427 estudiantes universitarios balanceados por sexo, a los cuales se les administró un cuestionario que indagaba acerca de conducta violenta en las relaciones de pareja. El 57 \% reporta haber vivido alguna vez en su vida violencia psicológica y $26 \%$ violencia física. Los factores asociados a recibir violencia física fueron: sexo, violencia psicológica recibida, actitudes favorables hacia la violencia, y baja participación religiosa, las variables asociadas a la violencia psicológica fueron: sexo, violencia física recibida, actitudes favorables hacia la violencia, y mayor tiempo de relación de pareja. Los resultados indican que la violencia de pareja es un problema significativo en los universitarios y sus características facilitan su invisibilización, dificultando un abordaje oportuno.

Palabras clave autores

Violencia de pareja, estudiantes universitarios.

Palabras clave descriptores

Estudiantes universitarios, Chile, violencia conyugal, aspectos psicológicos.

\section{A B S T R A C T}

The present study sought to estimate the magnitude of intimate partner violence (IPV) in university students in the southern region of Chile; to describe its manifestations, its associated factors, consequences and coping strategies. Method: A descriptive quantitative design was used, the sample was constituted by 447 university students randomly selected balanced by sex. Participants were asked about violent behaviour conduct through a questionnaire. Results: $57 \%$ of those questioned reported having experienced some psychological abuse, $26 \%$ reported physical violence at least once in their lifetime. Associated factors to receive physical violence were: sex, suffering psychological violence, favourable attitudes towards violence, and low religious participation. Associated factor to receive psychological violence was: sex, physical violence received, favourable attitudes towards the violence, and the length of the relationship. Discussion: Typical features of intimate partner violence in college life settings seem to favor its invisibility, thus turning it into a difficult phenomenon to approach and to prevent. Key words authors Intimate Partner Violence, University Students.

Key words plus

College Students, Conjugal Violence, Psychological Aspects, Chile. 


\section{Introducción}

La violencia íntima en jóvenes es un problema relevante por el impacto en la salud física y mental, y por el riesgo de que se transforme en un modelo estable de conducta con serias consecuencias para la vida conyugal y familiar ( $\mathrm{O}^{\prime}$ Leary, Barling, Arias, Rosenbaum, Malone \& Tyree, 1989; Pederson \& Thomas, 1992). Desde la década de 1980, numerosos estudios en Europa y Estados Unidos han mostrado que las cifras de violencia en parejas no casadas, es tan alta como en la parejas que cohabitan (González \& Santana, 2001; Strauss, 2004). Este tipo de violencia se define como abuso físico, emocional y sexual, en una relación romántica estable en que no existe vínculo legal ni cohabitación (Ruiz \& Fawcett, 1999). Algunas de las manifestaciones físicas de este fenómeno son: golpes, empujones, caricias violentas; en el plano emocional: insultos, humillaciones, descalificaciones, negación de la relación, y control sobre el otro respecto de sus vínculos familiares y sociales; y en el ámbito sexual: imponer contactos sexuales en contra de la voluntad, impedir el uso de anticoncepción y forzar a realizar prácticas sexuales indeseadas (Bookwala, Frieze, Smith \& Ryan, 1992; Canada Minister of Health, 1996).

La prevalencia de este tipo de violencia fluctúa entre 9 y $46 \%$; esta variación se relaciona con la falta de consenso respecto a las manifestaciones de la violencia, con el tipo de instrumentos de recolección de datos usado, con el periodo de tiempo reportado (violencia en el último año o violencia a lo largo de la vida) y con el tipo de población estudiada (Billigham, 1987; Bookwala et al., 1992; Glass, Fredland, Campbell, Yonas, Sharps \& Kub, 2003; Kann et al., 2000; Price, Byers, Sears, Whelan \& SaintPierre, 2000; Strauss, 2004; White \& Koss, 1991).

Entre los escasos estudios sobre este tema en Chile, Aguirre y García (1996) encontraron, en una muestra de universitarios, que el $51 \%$ de los encuestados reportó haber sufrido agresión psicológica y el $24 \%$, violencia física al menos una vez durante el último año. Por otra parte, la encuesta del Instituto Nacional de la Juventud realizada en el año 2000 en la ciudad de Santiago de Chile, indicó que $28 \%$ de los jóvenes reconoce haber sufrido violencia psicológica y 12,2 \% física, no existiendo mayor diferencia entre hombres y mujeres (Instituto Nacional de la Juventud, 2001). Este último punto coincide con la literatura internacional que señala que la violencia en esta población suele darse en forma bidireccional donde a mayor violencia ejercida hacia la pareja, existiría una mayor violencia recibida (Arias, Samios \& O'Leary, 1987; Matthews, 1984; Silverman, Ray, Mucci \& Hathaway, 2001; Servicio Nacional de la Mujer [SERNAM], 2004; Wekerle, Wolfe, Hawkins, Pittman, Glickman \& Lovald, 2001).

La relevancia de la violencia íntima en esta etapa evolutiva, radica en que un aumento gradual de este tipo de interacción tiene como consecuencia una normalización de estas conductas, siendo un factor predisponente de la violencia conyugal (O’Leary et al., 1989; SERNAM, 2004). Smith, White y Holland (2003), en un estudio longitudinal con universitarios, encontraron que la violencia sufrida en la adolescencia fue un fuerte predictor de la violencia, en la etapa universitaria.

Con respecto a los factores asociados se han descrito, entre otros: el aprendizaje de actitudes tradicionales en relación con los roles de género, la agresividad de la pareja que gatilla una respuesta más violenta, la falta de habilidades de comunicación y de resolución de conflictos, la necesidad de control de la pareja relacionada principalmente con celos de carácter crónico, y la violencia vivida dentro de la familia de origen (González \& Santana, 2001; Price et al., 2000).

Existe poca información en relación con el impacto de la violencia en los jóvenes. Algunos efectos descritos en quienes sufren la violencia son: trastornos depresivos, deterioro del sentimiento de seguridad, la autoestima y la confianza, sentimientos de culpa, aislamiento, bajo rendimiento académico y mayor riesgo de consumo abusivo de substancias (O’Keefe, Brockopp \& Chew, 1986; Singer, Anglin, Song \& Lunghofer, 1995). Las consecuencias para quienes ejercen violencia son: riesgo de ruptura de la relación, sentimientos de vergüenza, rechazo y condena social, así como el riesgo de repetir el modelo de interacción violenta en futuras relaciones (Glass et al., 2003). 
Considerando la alta incidencia de este fenómeno a nivel internacional y la escasa información que existe en Chile respecto del tema, la presente investigación tuvo como objetivos estimar la magnitud de la violencia de pareja en una muestra de estudiantes universitarios de la ciudad de Temuco, describir las formas en que se manifiesta, los factores asociados y las consecuencias.

\section{Método}

\section{Muestra}

El universo estuvo constituido por 7479 estudiantes de la Universidad de la Frontera, del cual se seleccionaron aleatoriamente los sujetos a través de un muestreo bietápico por conglomerados. En la primera etapa, se seleccionaron 18 de las 31 carreras pertenecientes a todas las facultades que componen la universidad. En la segunda etapa, se sortearon las asignaturas dentro de las carreras hasta alcanzar el tamaño muestral requerido, considerando una prevalencia de $20 \%$ y un error de muestreo entre 15 y $20 \%$, lo que dio un tamaño de muestra de 472 con un nivel de confianza de un $95 \%$. Se descartó el $5.3 \%$ de los casos porque no habían tenido pareja o porque los cuestionarios tenían información incompleta o incongruente. La muestra final quedo constituida por 427 estudiantes, $4.5 \%$ de la Facultad de Agronomía, $21 \%$ de la Facultad de Educación, 46.1 \% de la Facultad de Ingeniería y $28.4 \%$ de la Facultad de Medicina, siendo proporcional a la matrícula de cada facultad.

\section{Instrumentos}

Para evaluar las variables del estudio, se procedió a la construcción de un cuestionario ad hoc, tomando como referencia la literatura nacional e internacional (i.e. Güesmez, Palomino \& Ramos 2002; Strauss, 1979).

El cuestionario fue sometido al arbitrio de 3 jueces expertos, quienes evaluaron la validez de constructo del instrumento. Una vez incorporadas las sugerencias, se realizó una aplicación piloto a una muestra de 20 estudiantes de características similares a las de los participantes del estudio. Después de los dos procedimientos, se contó con la versión definitiva del instrumento, el cual se describe a continuación.

Para medir la Violencia Psicológica, se utilizó una escala de seis ítems, con una confiabilidad estimada por medio del coeficiente alfa de Cronbach, de .79. La escala tiene cuatro opciones de respuesta (nunca; 1 o 2 veces; 3 a 5 veces; 5 o más veces) a mayor puntaje mayor frecuencia de episodios de violencia psicológica:

1. He perdido contacto con amistades, familiares, compañeros de carrera para evitar que mi pareja se moleste.

2. Mi pareja a menudo critica mi aspecto físico, mi forma de ser.

3. Mi pareja desconfía y me acusa de infidelidad sin fundamento.

4. Mi pareja descalifica mis éxitos.

5. Mi pareja me ha insultado.

6. Mi pareja me hace estar en permanente tensión y haga lo que haga se irrita o me culpabiliza.

La Violencia Física fue medida a través de cinco ítems, con el mismo formato de respuesta que la escala de Violencia Psicológica (nunca; 1 o 2 veces; 3 a 5 veces; 5 o más veces). El coeficiente de confiabilidad alfa de Cronbach fue de .69. Puntajes elevados sugieren mayor frecuencia de violencia física:

1. Me ha tirado cosas o ha roto objetos durante una disputa o discusión.

2. Mi pareja me da pellizcos y/o empujones.

3. Mi pareja me ha dado cachetadas.

4. Mi pareja me ha dado patadas.

5. Mi pareja me ha dado puñetazos.

Originalmente se pretendió evaluar violencia sexual, sin embargo, los ítems creados para tal efecto fueron descartados, pues sus propiedades psicométricas resultaron inadecuadas.

Para evaluar Actitudes hacia la Violencia Íntima, se construyó una escala de 9 ítems, con forma- 
to de respuesta tipo Likert de 5 opciones (muy de acuerdo, de acuerdo, indiferente, en desacuerdo, muy en desacuerdo). Se estimó una confiabilidad alfa de Cronbach de .90 (Apéndice).

Por último, se incluyó un apartado para indagar acerca de antecedentes demográficos, participación en organizaciones de distinto tipo (social, política, religiosa etc.), experiencia de violencia en la familia de origen - donde se preguntó respecto de haber visto o escuchado violencia del padre a la madre y viceversa - y castigo físico de parte de padres o cuidadores, así como redes de apoyo e impacto de la violencia.

\section{Procedimiento}

Las investigadoras, aplicaron los instrumentos a los estudiantes en las salas de clases en forma colectiva, el tiempo promedio de aplicación fue de 35 minutos. Se leyeron las instrucciones estandarizadas y se respondieron dudas que surgieron en el momento. Como resguardos éticos se insistió en el carácter voluntario y anónimo de la encuesta y en la confidencialidad de las respuestas. Se ofreció a quienes se sintieran afectados por el contenido de las preguntas tomar contacto con las investigadoras para eventuales derivaciones. Si bien ningún alumno rechazó explícitamente la encuesta, los cuestionarios fueron revisados para descartar aquellos incompletos o con patrones de respuesta incongruente.

\section{Análisis Estadísticos}

Se realizaron análisis descriptivos y de frecuencias. Se aplicó la prueba $t$ de Student para comparar muestras independientes. Para establecer asociación entre las variables, se efectuaron pruebas de correlación de Pearson y Rho de Spearman en función del tipo de variable.

\section{Resultados}

Las características demográficas de la muestra se presentan en la Tabla 1. De los 427 estudiantes, 53 $\%$ declara tener pareja actual. En cuanto al tiempo de duración de la relación, 20.8 \% señala menos de 6 meses, $13 \%$ entre 6 meses y un año, $22.9 \%$ entre 1 y 2 años y $43.3 \%$ por más de 2 años, encontrándose que la distribución es similar en ambos sexos.

TABLA 1

Características de la muestra

\begin{tabular}{|c|c|c|}
\hline Variables & $N$ & $\%$ \\
\hline $\begin{array}{l}\text { Sexo } \\
\text { Hombres } \\
\text { Mujeres }\end{array}$ & $\begin{array}{l}230 \\
217\end{array}$ & $\begin{array}{l}51.0 \\
49.0\end{array}$ \\
\hline$\frac{\text { Edad }}{18-25}$ & $\begin{array}{c}407 \\
40\end{array}$ & $\begin{array}{c}91.0 \\
9.0\end{array}$ \\
\hline $\begin{array}{l}\text { Estado civil } \\
\text { Soltero } \\
\text { Conviviente } \\
\text { Otros }\end{array}$ & $\begin{array}{c}430 \\
11 \\
6\end{array}$ & $\begin{array}{r}96.0 \\
3.0 \\
1.0\end{array}$ \\
\hline $\begin{array}{l}\text { Etnia } \\
\text { Chileno } \\
\text { Mapuche } \\
\text { Otro }\end{array}$ & $\begin{array}{r}397 \\
37 \\
13\end{array}$ & $\begin{array}{r}89.0 \\
8.0 \\
3.0\end{array}$ \\
\hline $\begin{array}{l}\text { Procedencia } \\
\text { Urbana } \\
\text { Rural } \\
\text { No contesta }\end{array}$ & $\begin{array}{c}403 \\
42 \\
2\end{array}$ & $\begin{array}{r}90.0 \\
9.0 \\
1.0\end{array}$ \\
\hline $\begin{array}{l}\text { Nivel socioeconómico } \\
\text { Alto } \\
\text { Medio } \\
\text { Bajo_ }\end{array}$ & $\begin{array}{r}96 \\
282 \\
69\end{array}$ & $\begin{array}{l}22.0 \\
63.0 \\
15.0\end{array}$ \\
\hline $\begin{array}{l}\text { Religión } \\
\text { Católico } \\
\text { Evangélico } \\
\text { Sin Religión } \\
\text { Otros }\end{array}$ & $\begin{array}{r}289 \\
41 \\
98 \\
19\end{array}$ & $\begin{array}{r}65.0 \\
9.0 \\
22.0 \\
4.0\end{array}$ \\
\hline $\begin{array}{l}\text { Nivel de Participación } \\
\text { Religiosa } \\
\text { Alto } \\
\text { Medio } \\
\text { Bajo } \\
\text { Sin participación }\end{array}$ & $\begin{array}{r}36 \\
103 \\
161 \\
147\end{array}$ & $\begin{array}{r}8.0 \\
23.0 \\
36.0 \\
33.0\end{array}$ \\
\hline $\begin{array}{l}\text { Trabaja } \\
\text { Si } \\
\text { No }\end{array}$ & $\begin{array}{c}52 \\
395\end{array}$ & $\begin{array}{l}19.0 \\
81.0\end{array}$ \\
\hline
\end{tabular}

Fuente: elaboración propia 
En relación con antecedentes de violencia en la familia de origen, $11 \%$ señala que alguna vez vio o escuchó a su padre golpear a su madre, y $6 \%$ la madre al padre. Respecto de maltrato físico en la infancia, 11\% fue golpeado en forma severa, $64 \%$ en forma moderada y $25 \%$ declara que nunca lo golpearon.

\section{Magnitud}

Más de la mitad de la muestra (57\%) reporta haber sufrido episodios de violencia psicológica, 24\% declara que esto ha sucedido una o dos veces y $34.9 \%$ refiere que estos episodios han sucedido más de 3 veces. Las conductas que alcanzaron mayor prevalencia fueron: "Mi pareja a menudo critica mi aspecto físico, mi forma de ser", "Mi pareja me ha insultado", "Mi pareja desconfía de mí y me acusa de infidelidad sin fundamento". No se encontraron diferencias de género en la magnitud ni en el tipo de conducta.

Alrededor de un cuarto de la muestra (26\%) reporta haber vivido violencia física al menos una vez. De ellos 20\% corresponde a violencia moderada, empujar, dar palmadas, mientras que $6 \%$ corresponde a violencia grave como patadas o puñetes. Del total que ha vivido violencia, 12\% señala que esta situación le ha ocurrido con más de una pareja. No se encontraron diferencias de género en la frecuencia de las conductas.

\section{Factores Asociados}

Violencia Psicológica: De todos los factores estudiados, se encontró asociación con las siguientes variables:

- Sexo: los varones reportaron haber recibido levemente más violencia psicológica en comparación con las mujeres.

- Violencia física recibida: es decir aquellos que sufrieron violencia psicológica tendieron también a recibir violencia física.

- Actitudes favorables hacia la violencia: quienes sufrieron violencia psicológica tendieron a justificar el uso de la violencia en el contexto interpersonal.

- Mayor tiempo de relación de pareja: es decir quienes recibieron violencia psicológica tenían relación de pareja de más larga data.

Violencia física: se encontró asociación con las siguientes variables:

- Sexo: los varones reportaron haber recibido levemente más violencia física en comparación a las mujeres.

- Violencia psicológica recibida: quienes recibieron violencia física también tendieron a recibir más violencia psicológica.

- Actitudes favorables hacia la violencia: quienes recibieron violencia física, tendían a justificar el uso de la violencia en contexto interpersonal.

- Baja participación religiosa: quienes recibieron violencia física reportaron menor participación religiosa (Tabla 2).

TABLA 2

Factores asociados a recibir violencia

\begin{tabular}{|c|c|c|}
\hline Variables & $\begin{array}{l}\text { Violencia } \\
\text { Física }\end{array}$ & $\begin{array}{l}\text { Violencia } \\
\text { Psicológica }\end{array}$ \\
\hline Sexo & $\begin{array}{l}t=2.005 \\
p=.046\end{array}$ & $\begin{array}{c}t=3.336 \\
p=.001\end{array}$ \\
\hline $\begin{array}{l}\text { Violencia psicológica } \\
\text { recibida }\end{array}$ & $\begin{array}{l}r=.56 \\
p<.001\end{array}$ & - \\
\hline $\begin{array}{l}\text { Violencia física re- } \\
\text { cibida }\end{array}$ & & $\begin{array}{l}r=.56 \\
p<.001\end{array}$ \\
\hline $\begin{array}{l}\text { Actitudes favorables } \\
\text { hacia la violencia }\end{array}$ & $\begin{aligned} r & =.16 \\
p & =.001\end{aligned}$ & $\begin{array}{c}r=.18 \\
p<.001\end{array}$ \\
\hline $\begin{array}{l}\text { Tiempo de relación } \\
\text { de pareja. }\end{array}$ & 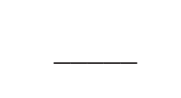 & $\begin{array}{c}\text { Rho }=.14 \\
p=.034\end{array}$ \\
\hline $\begin{array}{l}\text { Participación reli- } \\
\text { giosa }\end{array}$ & $\begin{array}{l}\text { Rho }=.10 \\
p=.035\end{array}$ & $\longrightarrow$ \\
\hline
\end{tabular}

Fuente: elaboración propia

No se encontró asociación entre violencia, ya sea psicológica o física, con los siguientes factores: estado civil, etnia, procedencia, nivel socioeconó- 
mico, persona con quien vive el estudiante, participación en actividades extraacadémicas, trabajo remunerado durante el periodo académico, violencia en la familia de origen, castigo físico en la infancia. Impacto

De los estudiantes que han sufrido violencia, $51 \%$ reporta haberse sentido afectado por esta situación. Las áreas con mayor impacto fueron: rendimiento académico $(21 \%)$, confianza en las relaciones de pareja (22\%), autoestima (17\%), salud mental (16 $\%)$. El análisis por género no muestra diferencias significativas en relación con las consecuencias.

La mayoría de los estudiantes señalan que la violencia recibida no fue causa de la terminación de la relación, dado que solo un $36 \%$ de los afectados dejó a la pareja por esa razón. Los argumentos citados para continuar la relación fueron: "porque la/lo quería" $48 \%$, "pensé que cambiaría" 18.7 \%, y porque "lo/la perdonó" $16.3 \%$. Un $14 \%$ de los sujetos plantea otras razones, siendo las más frecuentes la resolución del conflicto a través del dialogo, o que el episodio no fue suficientemente importante como para terminar definitivamente la relación. El análisis por género no mostró diferencias significativas.

Respecto de la búsqueda de ayuda $57 \%$ mantuvo esta situación en secreto, quienes compartieron esta situación, lo hicieron mayoritariamente con los amigos(as) (34\%), y en menor medida con sus familiares $(12,6 \%)$. Al respecto el análisis por género revela que las mujeres comparten más que los varones, diferencia que resultó estadísticamente significativa $(t=-2084 ; p<.05)$, además se encontraron diferencias en relación con las fuentes de apoyo; si bien las mujeres acuden en bajo porcentaje por ayuda a la familia de la pareja, ningún varón lo hace $(t=-2.120 ; p<.05)$. Un porcentaje mínimo de los estudiantes (4\%) recurre a instituciones de servicio público como centros de salud dado que muy pocos requirieron atención médica como consecuencia de la violencia, no apreciándose en este aspecto diferencias de género. Un hallazgo relevante es que quienes se sintieron más afectados tendieron a mantener en secreto esta situación $(t=3,598 ; p<.05)$.

\section{Discusión}

La prevalencia de Violencia Psicológica, expresada principalmente en insultos, críticas, desconfianza y control, alcanza un nivel superior a lo reportado en estudios similares, abarcando más de la mitad de la muestra. Esta diferencia sería esperable puesto que en la investigación de Aguirre y García (1996) se indaga sobre violencia en el último año, a diferencia de la presente investigación que reporta sobre la violencia recibida a lo largo de la vida. Por otra parte, es importante señalar que más de un tercio de la muestra reconoce que estos episodios habrían ocurrido tres o más veces, lo que podría constituir un grupo de riesgo en el cual la violencia está instalada como una pauta de interacción habitual, particularmente para quienes señalan que esto ha sucedido con más de una pareja (Smith et al., 2003).

La Violencia Física, que alcanza a un cuarto de la muestra, se expresa principalmente en conductas como pellizcos y empujones, siendo poco frecuentes las manifestaciones más graves como cachetadas y patadas, lo que se podría atribuir entre otros al control social ejercido por el contexto universitario que no legitima este tipo de conductas (SERNAM, 2004). Cabe destacar que estudios cualitativos en población universitaria, demuestran que algunas conductas agresivas tienen una connotación lúdica para los estudiantes, lo que reflejaría una tendencia a la minimización e invisibilización de la violencia (Aguirre \& García, 1996; González \& Santana, 2001; Póo \& Vizcarra, 2008).

La frecuencia levemente mayor de agresiones de parte de las mujeres, un hallazgo que resultó sorprendente para las autoras, pero que ha sido descrito frecuentemente en la literatura internacional (Straus, 2004; Glass et al., 2003), puede tener distintas explicaciones. Pederson y Thomas (1992) sugieren que en los varones habría un subreporte de estas conductas, mientras que las mujeres estarían más dispuestas a aceptar su responsabilidad, probablemente porque existe menor sanción social frente a la agresión femenina y ellas no percibirían su conducta como dañina. Este hallazgo es corroborado por Harris y Knight-Bohnhoff (1996), 
quienes encontraron que la agresión femenina es percibida tanto por hombres como mujeres, como menos grave que la masculina (Glass et al., 2003). En lo que coinciden la mayoría de los investigadores, es que el modelo abusador/abusada no se aplicaría a la población adolescente o universitaria (Archer, 2000; Bookwala et al., 1992), lo que daría cuenta de una distribución de poder más igualitaria en las relaciones intergénero (Salinas \& Arancibia, 2006).

La asociación entre violencia física y psicológica sugiere que ésta se presentaría como una escalada de conductas que se inician con la violencia psicológica (Aguirre \& García, 1996; Bookwala et al., 1992). Lo anterior se ve reafirmado por el hecho que quienes viven violencia tienden a presentar una actitud menos sancionadora, incorporándola como una estrategia válida de resolución de conflictos (Aguirre \& García, 1996; Riggs \& O’Leary, 1996; Ferrer, Bosh, Ramis, Torres \& Navarro, 2006). En cuanto al tiempo de pareja como factor de riesgo de la violencia, Laner y Thompson (1982), sugieren que la mayor profundidad en las relaciones conllevaría el derecho implícito a influir y controlar al otro.

De todas las variables analizadas, la participación religiosa, independiente de la confesión, resultó ser un factor protector de recibir violencia física. Es posible suponer que la religión imprime, en los jóvenes, valores y actitudes que excluyen conductas violentas, a la vez que la participación religiosa se constituye en una fuente de contención y apoyo que provee modelos de conducta prosocial.

El hecho que solo la mitad de la muestra que ha sufrido violencia reporte impacto, podría relacionarse con el tipo de agresión recibida, mayoritariamente de intensidad leve así como con la connotación lúdica atribuida a algunas conductas agresivas. Un aspecto preocupante, sin embargo, es que quienes se sienten más afectados mantienen esta situación en secreto. Una explicación posible es la necesidad, en las mujeres, de proteger la propia imagen, debido a la percepción de responsabilidad en las relaciones de abuso, ya que no serían capaces de "frenar" la situación, y/o hacerse respetar (SERNAM, 2004); y, en el caso de los varones, por la deseabilidad social que les impediría presentarse como víctima, dado que trasgrede las pautas del rol de género (Aguirre \& García 1996; Ashley \& Foshee. 2005; Dunham \& Senn, 2000).

Por otra parte, quienes sí solicitan ayuda recurren fundamentalmente a sus pares, recurriendo en menor medida a la familia y muy escasamente a fuentes formales como policía o servicios de salud. Esto sería esperable considerando el tipo de violencia recibida, en su mayoría leve y moderada y por el temor a perder su independencia (Ashley $\&$ Foshee, 2005).

La invisibilización de la violencia en los jóvenes universitarios, facilitaría que esta se constituya en una pauta estable de comportamiento. Si se asocia a la minimización y ausencia de redes de apoyo, es posible predecir que esta situación se puede constituir en un riesgo significativo para los jóvenes. Lo anteriormente expuesto fundamenta la importancia de realizar intervenciones tempranas (Wolfe et al., 2003), implementando programas educativos en el ámbito universitario que faciliten el reconocimiento de la violencia y el desarrollo de estrategias no violentas en la resolución de conflictos.

\section{Referencias}

Aguirre, A. M. \& García, M. (1996). Violencia prematrimonial: un estudio exploratorio en universitarios. (Tesis para optar al grado de Licenciado en Psicología), Universidad Católica de Valparaíso, Chile.

Archer, J. (2000). Sex differences in aggression between heterosexual partners: A meta-analytic review. Psychological Bulletin, 126 (5), 651-680.

Arias, I., Samios, M. \& O'Leary, K. (1987). Prevalence and correlates of physical aggression during courtship. Journal of Interpersonal Violence, 2(1), 82-90.

Ashley, O. \& Foshee, V. (2005). Adolescent help-seeking for dating violence: Prevalence, sociodemographic correlates, and sources of help. Journal of Adolescent Health, 36(1), 25-31.

Billigham, R. E. (1987). Courtship violence: The patterns of conflict resolution strategies across seven 
levels of emotional commitment. Family Relations, 36(3), 283-289.

Bookwala, J., Frieze, I. H., Smith, C. \& Ryan, K. (1992). Predictors of dating violence: A multivariate analysis. Violence y Victims, 7(4), 297-311.

Canada, Minister of Health. (1996). Dating violence; any age issue. Mental Health Unit. Health care and Issues Division on Family violence.

Dunham, K. T., \& Senn, C. Y. (2000). Minimizing negative experiences: Women's disclosure of partner abuse. Journal of Interpersonal Violence, 15, 251-261.

Ferrer, V., Bosch, E., Ramis, C., Torres, G. \& Navarro, C. (2006). La violencia contra las mujeres en la pareja: creencias y actitudes en jóvenes universitarios. Psicothema, 18(3), 359-366.

Glass, N., Fredland, N., Campbell, J., Yonas, M., Sharps, P. \& Kub, J. (2003). Adolescent dating violence: Prevalence, risk factors, health outcomes, and implications for clinical practice. Journal of Obstetric, Gynecologic, and Neonatal Nursing, 32(2), 227-237.

González, R. \& Santana, J. (2001). La violencia en parejas jóvenes. Psicothema, 13(1), 127-131.

Güesmez, A., Palomino, N. \&. Ramos, M. (2002). Violencia sexual y física contra las mujeres en el Perú. Estudio multicéntrico sobre la violencia de pareja y la salud de las mujeres. Lima: Flora Tristán/UPCH/ OMS.

Harris, M. B. \& Knight-Bohnhoff, K. (1996). Gender and aggression: Perceptions of aggression. Sex Roles, 35(1-2), 1-25.

Instituto Nacional de la Juventud. (2001). Tercera Encuesta Nacional de Juventud (Tomo 1, Informe resumido de resultados). Santiago de Chile: Autor.

Kann, L., Kinchen, S. A., Williams, B. I., Ross, J., Lowry, R., Grunbaum, J. O. \& Kolbe, L. (2000). Youth risk behavior surveillance. United States Surveillances Summaries.

Laner, M. \& Thompson, J. (1982). En Servicio Nacional de la Mujer (Ed.), Análisis de la violencia en las relaciones de pareja entre jóvenes. Santiago de Chile: Servicio Nacional de la Mujer.

Matthews, W. J. (1984). Violence in college couples. College Student Journal, 18, 150-159.
O'Keefe, M. K., Brockopp, K. \& Chew, E. (1986). Teen dating violence. Social Work, 31 (6), 465-468.

O'Leary, K. D., Barling, J., Arias, I., Rosenbaum, A., Malone, J. \& Tyree, A. (1989). Prevalence and stability of physical aggression between spouses: A longitudinal analysis. Journal of Consulting and Clinical Psychology, 57(2), 263-268.

Pederson, P. \& Thomas, C. D. (1992). Prevalence and correlates of dating violence in a Canadian university sample. Canadian Journal of Behavioral Science, 24(4), 490-501.

Póo, A. M. \& Vizcarra, M. B. (2008). Violencia de pareja en jóvenes universitarios. Terapia Psicológica, 26(1), 81-88.

Price, L., Byers, S., Sears, H., Whelan, J. \& Saint-Pierre, M. (2000). Dating Violence amongst New Brunswick Adolescents: A summary of two studies [Research Paper Series No. 2]. Fredericton: University of New Brunswick, Muriel McQueen Fergusson Centre for Family Violence Research.

Riggs, D. S. \& O'Leary, K. D. (1996). Aggression between heterosexual dating partners: An examination of a causal model of courtship aggression. Journal of Interpersonal Violence, 11 (4), 519-540.

Ruiz, G. \& Fawcett, G. (1999). Rostros y máscaras de la violencia en el noviazgo. México: IDEAME.

Salinas, P. \& Arancibia, S. (2006). Discursos masculinos sobre el poder de las mujeres en Chile. Sujetos y subjetividades. Última Década, 25, 65-90.

Servicio Nacional de la Mujer. (2004). Análisis de la violencia en las relaciones de pareja entre jóvenes. Santiago: Autor.

Silverman, J., Ray, A., Mucci, L. \& Hathaway, J. (2001). Dating violence against adolescent girls and associated substance abuse, unhealthy weight control. Journal of the American Medical Association, 286(5), 572-578.

Singer, M. I., Anglin, T. M., Song, L. Y. \& Lunghofer, L. (1995). Adolescent's exposure to violence and associated symptoms of psychological trauma. Journal of the American Medical Association, 273(6), 477-482.

Smith, P., White, J. \& Holland, L. (2003). A longitudinal perspective on dating violence among adolescent and college-age women. American Journal of Public Health, 93(7), 1104-1109. 
Strauss, M. A. (1979). Measuring intrafamily conflict and violence: The Conflict Tactics Scale. Journal of Marriage and the Family, 41 (1), 75-88.

Strauss, M. A. (2004). Prevalence of violence against dating partner by male and female university students Worldwide. Violence against Women, 10(7), 790-811.

Wekerle, C., Wolfe, D. A., Hawkins, D. L., Pittman, A., Glickman, A. \& Lovald, B. E. (2001). Childhood maltreatment, posttraumatic stress symptomatology, and adolescent dating violence: Considering the value of adolescent perceptions of abuse and a trauma mediational model. Development and Psychopathology, 13(4), 847-871.

White, J. W. \& Koss, M. P. (1991). Courtship violence: Incidence in a national sample of higher education students. Violence and Victims, 6(4), 247-256.

Wolfe, D., Wekerle, C., Scott, K., Straatman, A., Grasley, C. \& Reitzel-Jaffe, D. (2003). Dating violence prevention with At-Risk Youth: A controlled outcome evaluation. Journal of Consulting and Clinical Psychology, 71 (2), 279-91. 


\section{Apéndice}

\section{Escala de actitudes hacia la violencia}

¿En qué circunstancias consideras justificado el uso de la violencia en la pareja?

C.3A. Cuando un miembro de la pareja es infiel

C.3B. Cuando un miembro de la pareja descalifica al otro delante de terceras personas

C.3C. Cuando un miembro de la pareja descalifica al otro delante de su familia

C.3D. Cuando un miembro de la pareja insulta al otro

C.3E. El uso de la violencia no se justifica en ninguna circunstancia

C.3F. En parejas con poca educación

C.3G. Cuando uno o los dos miembros de la pareja tiene una historia personal de maltrato o ha sido testigo de violencia en la familia de origen.

C. $3 \mathrm{H}$. Cuando uno o los dos miembros de la pareja presenta alteraciones emocionales como por ejemplo impulsividad, ansiedad, depresión.

C.3I. Cuando uno o los dos miembros de la pareja presenta consumo abusivo de sustancias como alcohol y/o drogas.

C.3J. Cuando uno de los miembros de la pareja se niega a tener relaciones sexuales.

Las preguntas se contestan en una escala Likert de cinco ítems

Muy de acuerdo, acuerdo, ni acuerdo ni desacuerdo, desacuerdo, muy en desacuerdo. 